should study, immediately and as thoroughly as possible, those which are within our reach and are disclosed by Kohlhörster's experiment. The ionisation of gases in: a sealed vessel has been measured in our atmosphere up to an altitude of 9000 metres ; but it is necessary to repeat the experiment at several places on the earth, and to extend it up to the greatest altitudes reached in exploring balloons. The undertaking, it is true, will be costly; it devolves especially on the countries which have the greatest resources. I proposed, at the International Astronomical Congress, which met at Rome in May last, that there should be international co-operation for the complete study of the electrical phenomena of our atmosphere at great altitudes. The determination of the exact origin of these penetrating radiations is one of the most important problems confronting physical astronomy at the present time.

\title{
The Desensitising of Silver Bromide-Gelatin Plates.
}

\section{By. Dr. T. Slater Price.}

$\mathrm{IT}^{\mathrm{T}}$ is well known that the more sensitive a photographic plate is, the greater the care that has to be taken with respect to the actinic value of the light used in the dark room during the operation of development. The less the amount of light used, the more difficult it becomes to control the result; and it is therefore not to be wondered at that attempts have been made to modify the course of procedure in such a way that the exposed plate could be developed in a fairly good light. During the last few years various so-called " desensitisers" have been put on the market ; when the exposed plate is either treated with a solution of these before development, or when some of the desensitiser is added to the developer, the plate can safely be developed in a light which would otherwise give rise to very bad fogging.

At the recent Deuxième Congres de la Chimie Industrielle, M. A. Seyewetz gave an interesting account of the subject, and his paper has been published in Chimie et Industrie, I922, 8, 308-3II.

A. and L. Lumière and Seyewetz, in 1907 , were the first to notice that a silver bromide-gelatin plate becomes less sensitive when bathed in a solution of a developer such as diaminophenol, quinol, or pyrogallol. The loss in sensitivity varied slightly in different regions of the spectrum, but was most marked in the yellow and green. At a much later date, in 1920 , Lüppo-Cramer noticed that the desensitising action was much increased when sulphite was omitted from the developing solution, that is, when the developer was used in such a condition that it readily oxidised in the air. After immersion for a minute in a 0.05 per cent. solution of the developer the plate could be developed in yellow light without fogging. Such a method of desensitisation was insufficient, however, for orthochromatic and panchromatic plates, and moreover, the solutions underwent rapid alteration in the absence of sulphite.

Desensitisation only became a practical proposition when Lüppo-Cramer, in r92I, discovered the pronounced desensitising action of the azine dye, Phenosafranine, and also of other dyes belonging to the same class, on ordinary and panchromatic plates. Contrary to what one would at first suppose to be the case, these dyes do not owe their action to functioning as colour screens ; solutions of Phenosafranine transmit red and violet light, and yet they desensitise plates for these regions of the spectrum. Also, the violet safranines desensitise just as do the red safranines, although their absorption spectra are very different. These facts are very similar to those observed with sensitisers, and
Lüppo-Cramer has shown that certain optical sensitisers for one haloid salt of silver may act as desensitisers for other salts. For example, Erythrosin, Rhodamine B, Pinachrome, and Pinacyanol, which are the best sensitisers for chloride and bromide of silver, when used in very dilute solutions $(x: 20000)$ diminish the sensitivity of silver iodide-gelatin plates from 6 to 16 times ; Phenosafranine gives a reduction in sensitivity of about 40 times.

Lumière and Seyewetz have made investigations to see if there is any relation between the desensitising power of a substance and its chemical constitution. They have found, with the safranines, that the presence of the characteristic phenazine grouping,<smiles>c1ccccc1</smiles>

is insufficient in itself, and that amino-groups substituted in the benzene nuclei must also be present. Thus Neutral Red, which is a Eurhoidine having the formula

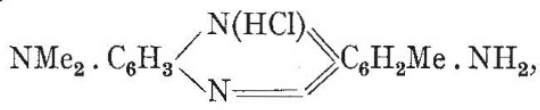

has desensitising properties approximating to those of Phenosafranine, which is<smiles>NCc1ccc(N)cc1</smiles>

Safranines in which one of the amino-groups has been eliminated, as in aposafranine, or in which this group is replaced by oxygen, as in the safranones, are notably less active as desensitisers. If both of the aminogroups are replaced by $\mathrm{O}$ or $\mathrm{OH}$, as in safranol, there is no longer any desensitising action. The acetylation of the amino-group, or its diazotisation and copulation with a phenol, destroys the desensitising properties of the safranine, while the replacement by ethyl of the phenyl group attached to the nitrogen has no effect. The Indulines, which are near cousins to the safranines, as also the thiazines and the oxazines, do not act as desensitisers. On the other hand, other colouring matters which have very different constitutions from that of safranine as, for example, picric acid, Indian Yellow, Chrysoidine, etc., are weak desensitisers ; Aurantia ( 1 : 1000) desensitises as actively as safranine for the blue rays, but is noticeably less active towards other parts of the spectrum.

It follows from the above that there does not seem 
to be a definite relation between the constitution of the dye and its desensitising properties. The conditions are thus very similar to those which hold in the case of sensitisers.

The great drawback to the use of Phenosafranine is its pronounced staining properties; it can only be removed from the gelatin film by prolonged washing. König has recently put on the market a desensitiser, Pinakryptol, which is claimed to be as active as Phenosafranine, but which neither stains gelatin, celluloid, nor the skin, although it gives a deep green solution.

A satisfactory explanation of the desensitising action of these substances is still wanting. Lüppo-Cramer claims that the phenomenon is connected with the formation of an oxidation product of the dye. Lumière and Seyewetz have shown, however, that if an unexposed plate is bathed in a solution of Phenosafranine it recovers its original sensitivity after being washed sufficiently long to remove the colouring matter. It is probable, according to Lumière and Seyewetz, that any oxidation product of the dye would be adsorbed by the silver bromide and not be removed by washing. so that the recovery in sensitivity would not be explicable on Lüppo-Cramer's theory.

\section{Obituary.}

Prof. Georges Lemoine.

$\mathrm{M}$ GEORGES LEMOINE, professor of chemistry at the Polytechnic School, Paris, whose death at the age of eighty-one has just been announced, was born at Tonnere in $\mathrm{r} 84 \mathrm{I}$. He entered the Polytechnic School in $185^{8}$, and two years later became Elève ingénieur at the École des Ponts-et-Chaussées. He early devoted himself to the study of chemistry, and investigated the compounds of sulphur and phosphorus, one of which, the sesquisulphide of phosphorus, is now largely employed in the igniting composition of the lucifer match in place of ordinary phosphorus. The substitution of this compound for phosphorus-now compulsory in most countries where matches are made - has been attended with the most beneficial results in the industry, the "phossy jaw" of the match-worker, or necrosis of the facial bones, being practically a thing of the past.

Lemoine also studied the reciprocal transformation of the two best-known allotropes of phosphorus. By heating known weights of phosphorus in closed flasks at $440^{\circ}$, the temperature of boiling sulphur, for varying lengths of time, and separating the products by carbon disulphide, he was able to determine the influence of time and pressure on the direction and extent of the change. He showed that the extent of the transformation is determined by the tension of the vapour, as in the case of other phenomena of volatilisation and dissociation. In vacuo, the conversion of ordinary into red phosphorus becomes more and more rapid as the temperature is raised. The rapidity of the transformation varies with the amount of phosphorus used. At any given moment the rapidity depends not only upon the quantity of ordinary phosphorus remaining, but also upon the quantity of red phosphorus already formed. The phenomenon is pre-eminently one of vapour tension and depends upon the capacity of the vessel in which the transformation-which is never complete-is effected. These facts are now well known and are uniformly acted upon in the phosphorus industry.

Questions of chemical dynamics had always a certain measure of attraction for Lemoine, and although he was not a particularly prolific contributor to chemical literature, much of his published work is concerned with their investigation. One of the most important of these inquiries relates to the conditions of chemical equilibrium of hydriodic acid. This substance was chosen as suitable for the study of the general phenomena of chemical equilibrium for the reason that the constituent elements are monatomic; they combine, or dissociate, without change of volume (at the temperature of the experiment), and the thermal effects of combination are very slight. The aim of the investigation was to show that under given conditions of temperature aid pressure, a mixture of the two constituent gases in given proportions will attain sooner or later a definite state of chemical equilibrium in which only a certain proportion of the hydriodic acid possible is actually formed, varying with the temperature, pressure, and proportions of the gases present, but always the same for the same conditions. The conditions studied were heat, pressure, mass, the action of porous bodies, of oxygen and of light. The main results have long since been incorporated into the general theory of chemical change, and call for no detailed account. At the time of their publication they constituted a notable and novel contribution to chemical dynamics.

It has long been known that mixed solutions of ferric chloride and oxalic acid are decomposed by light with the evolution of carbonic acid (Marchand, Jodin), and that the rate of decomposition depends on the intensity of the light. Lemoine studied this change with a view of determining how far it may be made the basis of an actinometric method. $\mathrm{He}$ found that for a given intensity, the evolution of gas is at first uniform, but that when about half the total quantity of carbon dioxide has been evolved, the rate of decomposition gradually diminishes. The greater the volume of the liquid, the longer is the time before decomposition slackens. When the two solutions are separately exposed to light for several hours and then mixed, decomposition takes place more rapidly than if the solutions had not been previously insolated. Dilution with water increases the change, due probably to hydrolysis of the ferric chloride. At ordinary temperatures the mixed solutions are practically unaffected in the dark. On heating, gas begins to be evolved at $50^{\circ}$ and increases rapidly in amount as the temperature rises. The general course of the change is, however, very similar to the influence exercised by light and is affected apparently by the same conditions.

Lemoine occasionally worked at subjects of organic chemistry, such as the nature of the paraffin hydrocarbons and the dissociation of haloid compounds of olefines under the influence of heat and pressure, but organic chemistry had evidently few attractions for him, and his work in this special field was very limited and calls for no special comment.

NO. 2773, VOL. I IO] 